\title{
Le Club français de la médaille et le musée du 11 Conti
}

The Club Français de la Médaille and the 11 Conti Museum

\section{Béatrice Coullaré}

\section{(2) OpenEdition}

\section{Journals}

Édition électronique

URL : http://journals.openedition.org/artefact/2459

DOI : $10.4000 /$ artefact.2459

ISSN : 2606-9245

Éditeur :

Association Artefact. Techniques histoire et sciences humaines, Presses universitaires du Midi

Édition imprimée

Date de publication : 6 décembre 2018

Pagination : 334-338

ISBN : 978-2-8107-0595-5

ISSN : 2273-0753

Référence électronique

Béatrice Coullaré, «Le Club français de la médaille et le musée du 11 Conti », Artefact [En ligne], 8 |

2018, mis en ligne le 21 juin 2019, consulté le 02 décembre 2020. URL : http://

journals.openedition.org/artefact/2459 ; DOI : https://doi.org/10.4000/artefact.2459

\section{(@) $\Theta \Theta \Theta$}

Artefact, Techniques, histoire et sciences humaines est mise à disposition selon les termes de la Licence Creative Commons Attribution - Pas d'Utilisation Commerciale - Pas de Modification 4.0 International. 


\section{Le Club français de la médaille et le musée du 11 Conti}

\section{Béatrice Coullaré}

\section{Résumé}

La Monnaie de Paris a une longue tradition de collaboration avec des artistes indépendants. Celle-ci a été facilitée par l'emploi du tour à réduire qui a permis aux modeleurs et aux sculpteurs de créer librement leurs œuvres sans devoir maîtriser la gravure en taille directe. Forte de son savoir-faire, c'est à partir de la seconde moitié du xxe siècle que la Monnaie de Paris s'est engagée dans un programme de valorisation de l'art de la médaille contemporaine grâce à la vision de Pierre Dehaye (1921-2008), son directeur. En poste depuis un an, il crée en 1963 le Club français de la médaille pour promouvoir et défendre cet art auprès des amateurs, des collectionneurs et du public. Grâce au dépôt légal, un exemplaire de chaque médaille frappée ou fondue pour ce club est entré dans les collections patrimoniales du musée du 11 Conti. Ce fonds est aujourd'hui valorisé par des expositions dossiers qui donnent à découvrir la richesse thématique et stylistique de cette collection unique.

\section{Mots-clés}

Médaille contemporaine, collection, musée, Monnaie de Paris, fonderie d'art, technique, savoir-faire, Pierre Dehaye

99 Béatrice Coullaré, «Le Club français de la médaille et le musée du 11 Conti », Artefact, 8, 2018, p. 334-338. 


\section{The Club Français de la Médaille and the 11 Conti Museum}

\section{Abstract}

The Monnaie de Paris has a long tradition of collaborating with independent artists. This was facilitated using the reducing machine which allowed modelers and sculptors to freely create their works without having to master the engraving in direct carving. With its know-how, it is from the second half of the $20^{\text {th }}$ century that the Monnaie de Paris embarked on a program to promote the art of the contemporary medal thanks to the vision of Pierre Dehaye (1921-2008), his director. In office for a year, he created in 1963 the Club français de la médaille to promote and defend this art among amateurs, collectors and the public. Thanks to the legal deposit, a copy of each coin struck or fused for this club has entered the patrimonial collections of the 11 Conti Museum. This collection is nowadays enhanced by exhibitions that showcase the thematic and stylistic richness of this unique collection.

\section{Keywords}

Contemporary medal, collection, museum, Monnaie de Paris, technique, knowhow, Pierre Dehaye 


\section{Les débuts de l'édition de médailles artistiques à la Monnaie de Paris}

La Monnaie de Paris travaillait déjà avec des artistes graveurs, médailleurs, modeleurs et sculpteurs indépendants ${ }^{1}$ au début du XIx ${ }^{e}$ siècle $^{2}$. Les relations entre ces derniers et l'institution se sont intensifiées et vraiment renforcées à partir des années 1880 quand la médaille a commencé à se démocratiser. Au tournant du $\mathrm{xx}^{\mathrm{e}}$ siècle, elle était pleinement reconnue comme un art majeur. L'usage généralisé du tour à réduire ${ }^{3}$, introduit dans les ateliers du quai de Conti en 1910 seulement, fut un facteur technique déterminant de ce succès, malgré les contestations de quelques détracteurs. En effet, ce procédé marqua un temps fort dans l'évolution esthétique de la médaille qui put se dégager des schémas et des canons conventionnels de l'art de la gravure en taille directe. Une confiance mutuelle s'était établie peu à peu entre les artistes et les mécaniciens, chargés de la mise au point des tours, dont la mission consistait à suivre le travail de gravure mécanisé des outillages de frappe. Ainsi déchargés d'une étape très délicate du travail, les artistes pouvaient se consacrer librement et entièrement à la création. Forte de cette expérience, la Monnaie de Paris se positionna durablement dans le paysage de l'édition de médailles artistiques à fortes valeurs décoratives. En 1938, elle franchit une étape décisive en créant la Fédération internationale des éditeurs de médailles ${ }^{4}$, en partenariat avec la Belgique, la Suisse et la Grande-Bretagne. Ce groupement de professionnels avait pour objectif principal de traiter des questions relatives au développement industriel de la médaille artistique.

\footnotetext{
1. Aujourd'hui des artistes viennent en résidence, dans le cadre des expositions d'art contemporain montées par la Monnaie de Paris, pour y découvrir les techniques de création et de fabrication des médailles.

2. Le monopole de la frappe, instauré par l'arrêté du 5 germinal an XII (26 mars 1804) resta en vigueur jusqu'en 1893. Les artistes et les éditeurs de médailles n'avaient pas d'autre choix que de faire frapper leurs médailles dans les ateliers de la Monnaie de Paris.

3. Ce procédé de gravure mécanique est toujours utilisé de nos jours car il est capable de reproduire un modèle dans les moindres détails, en respectant la sensibilité plastique des artistes.

4. Cette association à but non lucratif est toujours active de nos jours. Voir le site www.fidemmedals.org.
} 


\section{Pierre Dehaye, un visionnaire au service de la médaille contemporaine}

Après la Seconde Guerre mondiale, la Monnaie de Paris relance sa production de médailles décoratives ${ }^{5}$ en enrichissant régulièrement son catalogue général de nouvelles créations ${ }^{6}$. Mais cela ne suffit pas à pérenniser l'outil industriel. Il faut aller plus loin et engager une politique d'avenir pour ce secteur d'activité secondaire face à la production monétaire, en pleine expansion. C'est alors que son nouveau directeur, Pierre Dehaye, a l'idée de créer en 1963 le Club français de la médaille pour promouvoir et défendre la création de médailles artistiques contemporaines auprès des amateurs, des collectionneurs et du public. Son but est de soutenir les artistes par l'édition de médailles frappées (Fig. XXII, cahier couleur) et fondues (Fig. XXIII, cahier couleur) et de créer une fonderie d'art dans laquelle des œuvres inédites numérotées seront produites. Par ces créations originales, qui concernent aussi d'autres catégories d'objets tels que les jetons de vœux, les médailles calendriers et les plaques de cheminée, il s'agit de perpétuer la tradition de la gravure en taille directe, d'encourager l'usage du tour à réduire, de développer de nouvelles techniques de fonte d'art et de faire valoir le savoir-faire des fondeurs, des ciseleurs et des patineurs. Il s'agit, en d'autres termes, de mettre en lumière les métiers d'art de la Monnaie de Paris sans avoir "l'esprit de boutique ", comme le dit Pierre Dehaye lui-même dans un de ses écrits ${ }^{8}$. Pour plus de visibilité, l'action du club est relayée dès le mois de novembre 1963, par une revue intitulée Le Club français de la médaille puis Métal pensant, à partir de 1987. Des articles sur l'histoire de France de l'Antiquité à nos jours, sur l'histoire de l'art monétaire et de la médaille, sur les artistes et leurs rapports avec la Monnaie de Paris y sont publiés tous les trimestres. Ce club, qui a existé jusqu'au début des années 1990, a rencontré un énorme

5. Ne pas confondre cette production avec les décorations civiles et militaires.

6. Ce catalogue est encore aujourd'hui enrichi des nouvelles créations développées par le service du Marketing de la Monnaie de Paris.

7. Directeur de la Monnaie de Paris de 1962 à 1984. Élu membre de l'Académie des beaux-arts en 1975, Pierre Dehaye reçut de nombreuses distinctions honorifiques.

8. Pierre Dehaye, "Éditorial ", Le Club français de la médaille, bulletin n ${ }^{\circ}$ 1, 1963, p. 3. Du même auteur dans la même revue, "Trois ans déjà », n 13, 1966, p. 5 ; « Dix ans ", n 41, 1973, p. 7-13; «Éditorial, vingt ans! », n 81, 1983, p. 4. 
succès ${ }^{9}$. De ce point de vue, Pierre Dehaye fut un visionnaire qui n'eut pas d'égal parmi les directeurs d'institution monétaire à la même époque. On peut dire que l'art de la médaille contemporaine lui doit tout.

\section{La valorisation du fonds du Club français de la médaille au musée du 11 Conti}

Grâce au dépôt légal ${ }^{10}$, les collections patrimoniales de la Monnaie de Paris conservent un exemplaire de chaque pièce éditée par le Club français de la médaille. Ce fonds forme aujourd'hui la part la plus importante des collections d'art contemporain du musée du 11 Conti. Son catalogue, doté de près de cinq cents noms d'artistes contemporains français et étrangers forme un des " annuaires " les plus atypiques et originaux de la création artistique des années 1960 à 1990. La grande diversité des thèmes abordés, la richesse stylistique de chaque courant ou école offre un catalogue inépuisable de sujets d'expositions dossiers qui permettent de faire découvrir au public une large palette de talents et de formes. À titre d'exemple, dans le sillage de l'exposition consacrée au plasticien indien Subodh Gupta, une exposition dossier intitulée "Un rêve d'ailleurs, l'art de la médaille et l'Inde " (13 avril-28 octobre 2018) a permis d'exposer une sélection de médailles, éditées au fil des ans pour les adhérents du club, qui permettent d'évoquer ce pays. D'autres expositions du même genre permettront, dans les années à venir, de révéler au public la richesse inestimable de ce fonds de médailles contemporaines.

9. Vingt ans après sa création, soit en 1983, le club comptait près de 8000 membres français et étrangers.

10. Se reporter au Code des instruments monétaires et des médailles. 